\title{
Observation of Fermi-Pasta-Ulam-Tsingou Recurrence and Its Exact Dynamics
}

\author{
D. Pierangeli, ${ }^{1,2,3,}$ M. Flammini, ${ }^{2}$ L. Zhang, ${ }^{1}$ G. Marcucci, ${ }^{2,3}$ A. J. Agranat, ${ }^{4}$ \\ P. G. Grinevich, ${ }^{5}$ P. M. Santini, ${ }^{2,6}$ C. Conti, ${ }^{2,3}$ and E. DelRe ${ }^{2,3}$ \\ ${ }^{1}$ International Collaborative Laboratory of 2D Materials for Optoelectronic Science \& Technology, \\ Key Laboratory of Optoelectronic Devices and Systems of Ministry of Education and Guangdong \\ Province, College of Optoelectronic Engineering, Shenzhen University, 518060 Shenzhen, China \\ ${ }^{2}$ Dipartimento di Fisica, Università di Roma "La Sapienza”, 00185 Rome, Italy \\ ${ }^{3}$ ISC-CNR, Università di Roma "La Sapienza", 00185 Rome, Italy \\ ${ }^{4}$ Applied Physics Department, Hebrew University of Jerusalem, 91904 Jerusalem, Israel \\ ${ }^{5}$ L.D. Landau Institute for Theoretical Physics, 142432 Chernogolovka, Russia \\ ${ }^{6}$ INFN-Istituto Nazionale di Fisica Nucleare, Sezione di Roma, 00185 Rome, Italy
}

(Received 8 June 2018; revised manuscript received 2 September 2018; published 29 October 2018)

\begin{abstract}
One of the most controversial phenomena in nonlinear dynamics is the reappearance of initial conditions. Celebrated as the Fermi-Pasta-Ulam-Tsingou problem, the attempt to understand how these recurrences form during the complex evolution that leads to equilibrium has deeply influenced the entire development of nonlinear science. The enigma is rendered even more intriguing by the fact that integrable models predict recurrence as exact solutions, but the difficulties involved in upholding integrability for a sufficiently long dynamic has not allowed a quantitative experimental validation. In natural processes, coupling with the environment rapidly leads to thermalization, and finding nonlinear multimodal systems presenting multiple returns is a long-standing open challenge. Here, we report the observation of more than three Fermi-Pasta-Ulam-Tsingou recurrences for nonlinear optical spatial waves and demonstrate the control of the recurrent behavior through the phase and amplitude of the initial field. The recurrence period and phase shift are found to be in remarkable agreement with the exact recurrent solution of the nonlinear Schrödinger equation, while the recurrent behavior disappears as integrability is lost. These results identify the origin of the recurrence in the integrability of the underlying dynamics and allow us to achieve one of the basic aspirations of nonlinear dynamics: the reconstruction, after several return cycles, of the exact initial condition of the system, ultimately proving that the complex evolution can be accurately predicted in experimental conditions.
\end{abstract}

DOI: 10.1103/PhysRevX.8.041017

Subject Areas: Nonlinear Dynamics, Optics, Statistical Physics

\section{INTRODUCTION}

Nonlinear interaction in a multimodal system introduces coupling between its linear modes. When a reduced set of modes is initially excited, the energy exchange associated with this coupling provides the route to reach thermodynamic equilibrium. However, as discovered by Fermi in collaboration with Pasta et al. [1], the irreversible process towards thermalization can present local reversibility. Studying a chain of anharmonic oscillators with a singlemode initial condition, they found that the system fails to thermalize on small timescales and undergoes a dynamics

\section{*Davide.Pierangeli@roma1.infn.it}

Published by the American Physical Society under the terms of the Creative Commons Attribution 4.0 International license. Further distribution of this work must maintain attribution to the author(s) and the published article's title, journal citation, and DOI. characterized by the quasiperiodic appearance of specific states, a behavior known as Fermi-Pasta-Ulam-Tsingou (FPUT) recurrence [2-4].

An approach to understanding the physical mechanism underlying the phenomenon rests on the quasi-integrability of the system [5-8]. This property implies the existence of a timescale for which the FPUT dynamics is essentially integrable. In fact, for integrable models, pure thermalization is never reached since normal modes are phase locked and not free to resonantly interact and spread energy over the entire spectrum [6]. Consistently, certain integrable systems support breathers; their phase space presents homoclinic orbits connecting unstable solutions, so trajectories starting in the proximity of these unstable points can return close to the original state.

The key role of integrability explains why the FPUT recurrence has eluded in-depth experimental investigations. Specifically, while in numerical studies the thermalization time was too large to be initially identified, quite the opposite 
issue arises in experiments: In open systems involving several interacting modes, recurrences to the initial state are not normally reported. In fact, because of the effect of intrinsic dissipation or input noise amplification [9], a natural process is rarely integrable and does not preserve multiple returns. Observations in quasi-Hamiltonian systems have so far been limited to one or two return cycles [10]. Evidence of the recurrence of states has been reported in deep water waves [10], surface gravity waves [11], magnetic rings [12], optical microresonators [13], and optical fibers [14-16]. In spite of these efforts, how the specific initial condition determines the properties of the recurrent behavior remains a fundamental point that has never found experimental validation. An important attempt in this direction has been reported very recently in loss-compensated optical fibers [16], where, however, the tailored amplification only allows the system to mimic the return cycles that would have its nondissipative counterpart. In this setting, among the many recurrent behaviors expected by varying the input state, only the two types with opposite symmetry have been observed and related to separate families of orbits in phase space [16]. The observation of the FPUT dynamics as predicted by exact solutions of an underlying integrable model remains an open challenge.

Here, we observe the Fermi-Pasta-Ulam-Tsingou recurrence in spatial nonlinear optics and provide evidence that the recurrent behavior is governed by the exact solution of the nonlinear Schrödinger equation (NLSE) integrable dynamics. Specifically, we exploit a three-wave interferometric setup to finely tune the amplitude and phase of the single-mode input excitation propagating in a photorefractive medium. The unstable mode manifests the Akhmediev breather $(\mathrm{AB})$ profile and undergoes several growth and decay cycles whose partial period and phase shift are determined by the initial excitation, in remarkable agreement with the analytic nonlinear Schrödinger theory. This allows us to retrieve the specific input state from the properties of the nonlinear stage of instability, the signature of the predictability of the underlying dynamics.

\section{EXACT RECURRENCES IN THE NONLINEAR SCHRÖDINGER EQUATION}

The integrable NLSE,

$$
i \partial_{z} \psi+\partial_{x x} \psi+2|\psi|^{2} \psi=0,
$$

is a universal model describing the propagation of a quasimonochromatic field $\psi(x, z)$ in a weakly nonlinear medium [17]. Exact solutions of Eq. (1) corresponding to perturbations of the constant background wave have recently attracted considerable attention in hydrodynamics and optics [18-24], in particular, in the way they describe the dynamics of the modulation instability (MI) and may be relevant in explaining the formation of extreme amplitude waves (rogue waves) [25-30]. On the other hand, the NLSE naturally arises as the continuous limit (infinite number of modes) for the dynamics of a chain of anharmonic oscillators coupled by a cubic nonlinearity, the so-called $\beta$-FPUT model $[31,32]$. In this framework, the problem of finding the timescale of the recurrence as a function of the specific input condition has been elusive until recently. The analytic description of the recurrence for an initially perturbed background field of finite length with a single unstable mode has been reported by Grinevich and Santini using the finite-gap method or matched asymptotic expansions $[33,34]$. Theory points out a variety of phase-shifted recurrences closely determined by the phase and amplitude of the input condition. In particular, considering the singlemode perturbed input field

$$
\psi_{0}(x)=1+\varepsilon\left(c_{1} e^{i k x}+c_{2} e^{-i k x}\right),
$$

with complex amplitudes $c_{1}$ and $c_{2}$ and $\varepsilon \ll 1$, we expect the recurrent growth of a coherent structure of the Akhmediev type ( $x$ periodic) and its recurrent decay to the initial state. The first-appearance time or recurrence partial period of this large-amplitude wave is predicted to be $[33,34]$

$$
Z_{1}=\frac{1}{\sigma_{k}} \log \left(\frac{\sigma_{k}^{2}}{2 \varepsilon|\alpha|}\right)
$$

where $\sigma_{k}=k \sqrt{4-k^{2}}$ is the growth rate of the input unstable mode with wave vector $k$ and $\alpha=c_{1}^{*}-$ $c_{2} \exp (2 i \vartheta)$ with $\vartheta=\arccos (k / 2)$. The multiple recurrence of the field to the initial condition corresponds to periodic orbits close to the homoclinic orbit described by the wellknown AB exact solution of the NLSE [35]. In fact, in the $m$ th recurrent nonlinear stage of the dynamics $(m \geq 1)$, the field is described by the $\mathrm{AB}$ soliton, which, at its maximum, reads as

$\psi\left(x, Z_{m}\right)=e^{i \xi_{m}} \frac{\cos (2 \vartheta)+\sin (\vartheta) \cos \left[k\left(x-X_{m}\right)\right]}{1-\sin (\vartheta) \cos \left[k\left(x-X_{m}\right)\right]}+O(\varepsilon)$,

where $\xi_{m}, Z_{m}$, and $X_{m}$ are parameters related to the input condition through the elementary functions $[33,34]$

$$
\begin{aligned}
& Z_{m}=Z_{1}+(m-1) \frac{2}{\sigma_{k}} \log \left(\frac{\sigma_{k}^{2}}{2 \varepsilon \sqrt{|\alpha \beta|}}\right), \quad m \geq 1, \\
& X_{m}=\frac{\arg (\alpha)-\vartheta+\pi / 2}{k}+(m-1) \frac{\arg (\alpha \beta)}{k}, \\
& \xi_{m}=2 Z_{m}+2(2 m-1) \vartheta,
\end{aligned}
$$

with $\beta=c_{2}^{*}-\exp (-2 i \vartheta) c_{1}$. Although solutions of the Akhmediev type have been observed and connected to recurrent behaviors in different settings $[10,16]$, experimental demonstration of Eq. (3), which forms the basis for 
the FPUT dynamics in a broad range of systems, is lacking. In other words, the way in which these exact recurrent solutions can have physical relevance is an open question.

\section{SPATIAL OPTICAL SETTING}

To investigate FPUT recurrences in optical dynamics, we consider the propagation of nonlinear optical waves in a photorefractive crystal. The wave vectors of the optical field constitute the linear modes that are coupled by nonlinear propagation. The transverse crystal size fixes the finite length of the input wave, a condition ensuring a countable set of Fourier modes and a finite recurrence period. Under specific conditions, the system can be described by the NLSE in the spatial domain, with the propagation direction acting as an evolution coordinate [36]. The experimental geometry of our setup is shown in Fig. 1(a) and detailed in the Appendix. Three continuous laser sheets are made to symmetrically interfere at the input facet of a potassium-lithium-tantalate-niobate (KLTN) crystal to form a quasi-one-dimensional background wave with a coherent single-mode perturbation. Along the transverse $x$ direction, the relevant one for the dynamics under study, the optical field resulting from the symmetric interference of three mutually coherent, linearly polarized optical waves is $E=E_{0}+E_{1} e^{i \phi_{1}} e^{i k x}+E_{2} e^{i \phi_{2}} e^{-i k x}$, with $k=2 \pi \tan (\theta) / \lambda$. The optical intensity normalized to the background $I / I_{0} \quad\left(I_{0}=\left|E_{0}\right|^{2}\right)$ can be expressed as

(a)
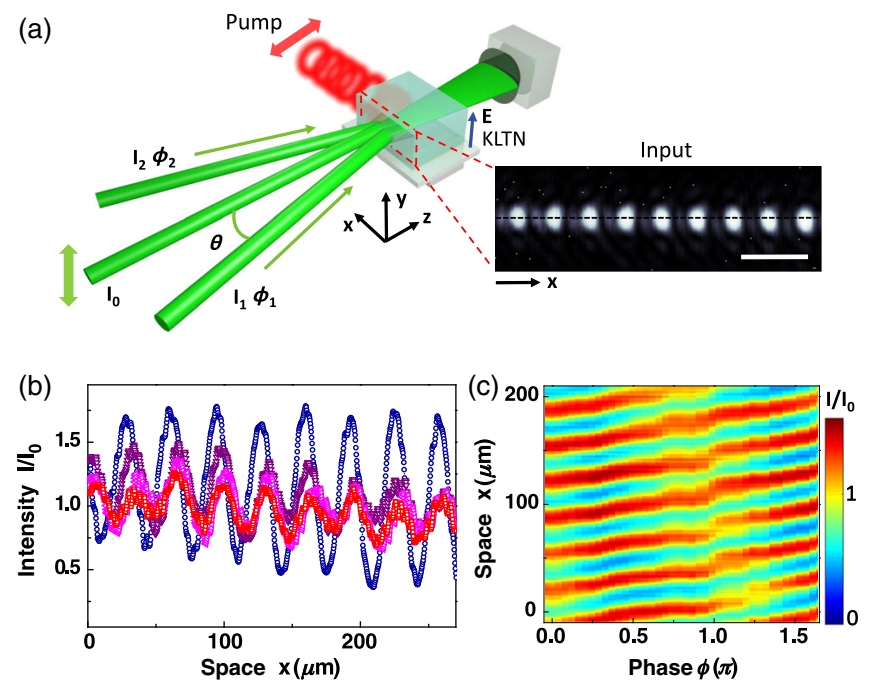

FIG. 1. Experimental setup. (a) Sketch for the symmetric threewave interferometric scheme used to generate a quasi-onedimensional background wave with a single-mode perturbation that propagates in a pumped photorefractive KLTN crystal (see the Appendix). The inset shows an example of the detected input intensity distribution (scale bar is $50 \mu \mathrm{m}$ ). (b) Input intensity $x$ profiles normalized to the background for different amplitudes of the harmonic perturbation $\left(k=0.019 \mu \mathrm{m}^{-1}\right)$. (c) Phase control of the initial condition: intensity distribution varying the relative phase $\phi$ between the interfering waves.
$I / I_{0} \equiv\left|\psi_{0}(x)\right|^{2}=1+A \cos (k x+B)$, which directly maps the initial condition in Eq. (2) with $A=2 \varepsilon|\gamma|, B=\arg (\gamma)$, and $\gamma=c_{1}+c_{2}^{*}$. With respect to the experimentally accessible parameters, the amplitude and phase of the perturbation read as $A=2 \sqrt{\left[I_{1}+I_{2}+2 \sqrt{I_{1}} \sqrt{I_{2}} \cos \left(\phi_{1}+\phi_{2}\right)\right] / I_{0}}$ and $\tan (B)=\left(\sqrt{I_{1}} \sin \phi_{1}-\sqrt{I_{2}} \sin \phi_{2}\right) /\left(\sqrt{I_{1}} \cos \phi_{1}+\sqrt{I_{2}} \cos \phi_{2}\right)$. Therefore, the spatial frequency of the perturbation $k$ can be varied by acting on the geometrical angle $\theta$ in between the arms of the interferometer, whereas their optical power and phase delay $\phi \equiv \phi_{1}+\phi_{2}=\arg \left(c_{1}\right)+\arg \left(c_{2}\right)$ set, respectively, the amplitude and phase of the single mode [Figs. 1(b) and 1(c)]. The fringe visibility is thus maximum for $\phi_{1}+$ $\phi_{2}=0$ and minimum for $\phi_{1}+\phi_{2}=\pi$. In the symmetric case $I_{1} \simeq I_{2}$, we have $B \simeq\left(\phi_{1}-\phi_{2}\right) / 2$.

Since the propagation length cannot be varied in our setting and the intensity profile inside the crystal cannot be directly measured [37], nonlinear evolution of the input field is observed by the time the crystal is exposed to the copropagating light beams. The method relies on the nature of the photorefractive nonlinearity, which is noninstantaneous and accumulates in time as a photogenerated spacecharge field builds up [38]. Since the process occurs on a slow timescale compared to wave propagation through the medium, this implies a nonlinear coefficient that depends parametrically on the exposure time. Because of the invariant properties of the wave equation, observations of the intensity distribution at the crystal output at different times correspond to beam propagation for increasing effective distances $Z$ (see the Appendix). This is equivalent to studying the dynamics by varying the strength of the nonlinearity through an external parameter, in close analogy with FPUT investigations in optical fibers where changes of the input optical power are exploited [14].

\section{EXPERIMENTAL RESULTS}

The spatial intensity distribution $I(x) / I_{0}$ detected as a function of the evolution coordinate $Z$ is reported in Fig. 2(a) for $k=0.019 \mu \mathrm{m}^{-1}, A=0.3$. We observe the input perturbation growth on the modulationally unstable background forming a train of large-amplitude localized waves, which decays back to an almost constant background and recurrently reappears from it. The set of linear modes undergoes several return cycles in which energy flows back and forth, passing from the zero and first modes (the initial perturbation) to a spectral distribution in which all the modes are excited, the signature of the FPUT dynamics [39]. At variance with classical and quantum beating, such as Rabi cycles in two-level quantum systems [40], here energy oscillations involve several modes and occur without any driving field. At each cycle, the whole field distribution is spatially shifted by an amount $\Delta$, a phenomenon also referred to as broken symmetry of FPUT recurrence $[10,16]$. Although a similar phase shift has been theoretically associated with the specific gain of 

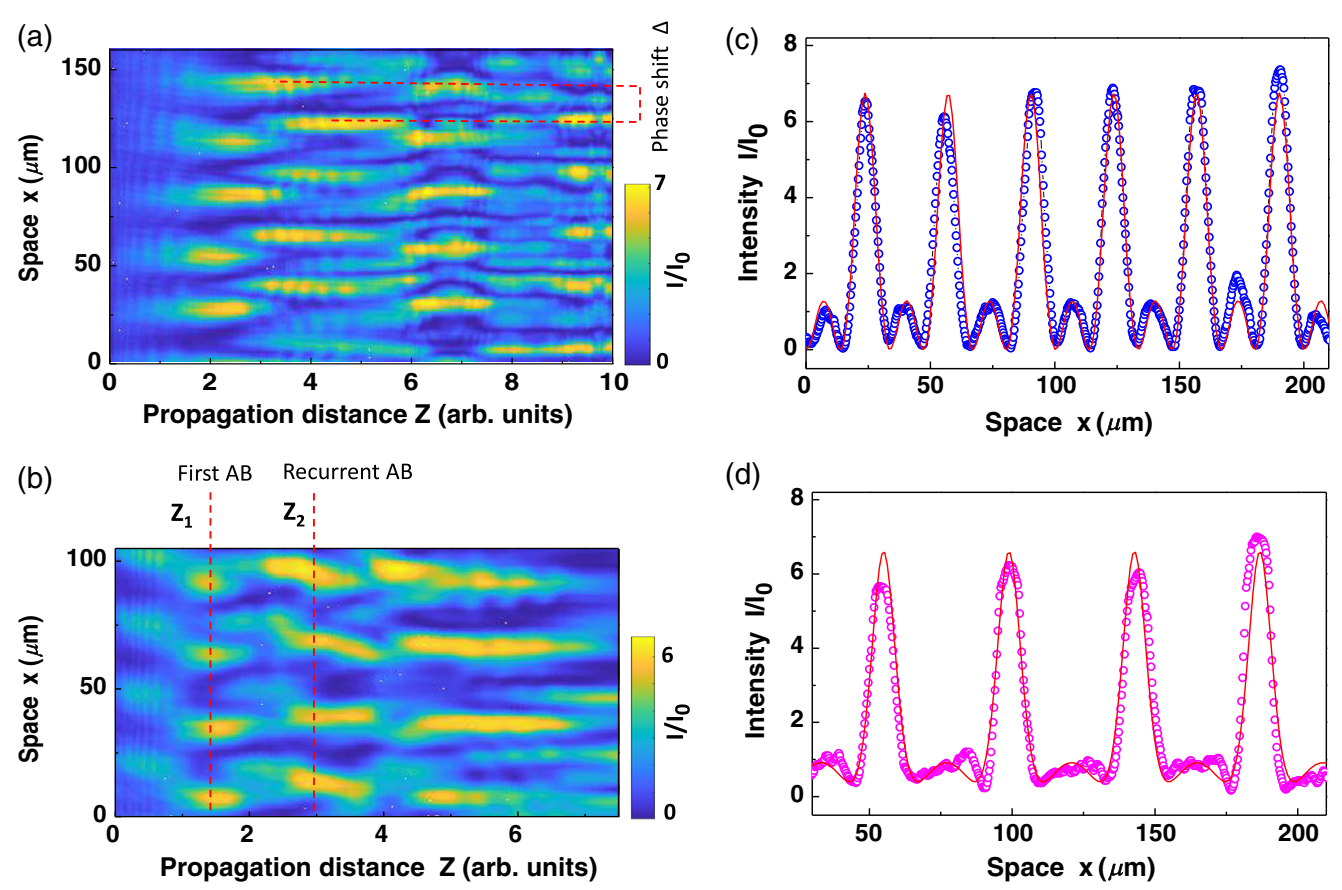

FIG. 2. Observation of the FPUT recurrence of Akhmediev breathers. (a,b) Evolution of the detected spatial intensity distributions for (a) $k=0.019 \mu \mathrm{m}^{-1}, A=0.3$ and (b) $k=0.030 \mu \mathrm{m}^{-1}, A=0.5$. Both observations show the appearance of a high-intensity pattern at a distance $Z_{1}$ (red dotted line), its return to the initial state, and multiple recurrences with a spatial phase shift that depends on the experimentally assigned input condition $[\Delta \approx 15 \mu \mathrm{m}$ and $2 \mu \mathrm{m}$ in panels (a) and (b), respectively]. (c,d) Intensity $x$ profile measured at the first appearance of the localized waves (circles) fitted with the AB profile at its maximum [red line, Eq. (4)] for (c) $k=0.021 \mu \mathrm{m}^{-1}$ and (d) $k=0.014 \mu \mathrm{m}^{-1}$.

the seeded wave vector [41] and the effect of dissipation [42], we show hereafter that it results from the sensitivity of the dynamics to the specific initial phase. This phase shift, as well as the recurrence period detected through the first appearance distance $Z_{1}$ of the high-intensity pattern, strongly changes as the input perturbation is varied. For instance, in Fig. 2(b), we report the observed FPUT recurrence for $k=0.030 \mu \mathrm{m}^{-1}$ and $A=0.5$, where no significant phase shift occurs. The recurrent behavior can be directly related to the excitation from the single-mode input perturbation of an orbit close to the Akhmediev breather [34]. As shown in Figs. 2(c) and 2(d), the periodic intensity profile detected along $x$ when the amplified modes reach their first maximum is well fitted by the Akhmediev breather solution of the NLSE at its maximum [Eq. (4)]. Consistently, throughout the paper, we refer to these localized states as $\mathrm{AB}$. The finding of exact solutions indicates that our system remains close to the integrable regime on these effective distances; that is, it can be properly described by the NLSE.

We study the FPUT recurrence by varying the singlemode input condition. Fixing the initial phase of the field through a careful maximization of fringe visibility, we first analyze the recurrence partial period by varying the amplitude of the perturbation $A$. The results in Fig. 3(a) show that the first appearance of the $\mathrm{AB}$ occurs at a distance that decreases as the single-mode amplitude becomes larger. An analogous behavior is observed for the recurrent breather (second appearance). In remarkable agreement with the analytic solution of the NLSE, the observed scaling follows Eq. (3), which predicts $Z_{1} \propto \log (1 / A)$. For modes $k$ falling in the proximity of the maximum gain, the recurrence period only weakly depends on the input wave vector [Fig. 3(b)], a feature well captured by Eq. (3) through $\sigma_{k}$. More importantly, the main effect on the recurrence is found to be related to the phase of the initial condition. To investigate its role, we balance the optical power in the interferometer arms $\left(I_{1} \approx I_{2}\right)$ and introduce a slight tilt in one of them, so as to have a perturbation with a phase that depends on the spatial point. The observed FPUT dynamics is reported in Fig. 3(c); the AB appears and recurs phase shifted at a propagation distance that varies along the transverse coordinate. As a function of the input phase delay, $Z_{1}$ presents an oscillation that has a sharp maximum for $\phi \simeq-0.3 \pi$ and a broad minimum for $\phi \simeq-0.9 \pi, 0.35 \pi$ [Fig. 3(d)]. This characteristic behavior, which reflects phase dynamics in each return cycle, is in remarkable agreement with the NLSE theory and represents its main validation. In fact, in Eq. (3), the recurrent semiperiod critically depends on $|\alpha|$, a quantity that oscillates with the relative phase of the complex amplitudes $c_{1}$ and $c_{2}$ forming the initial perturbation. Specifically, in the case of symmetric perturbations $\left(I_{1} \approx I_{2}\right)$, theory predict a sharp maximum in $Z_{1}$ for $\phi \simeq-2 \vartheta$; from Fig. 3(d), we can thus 

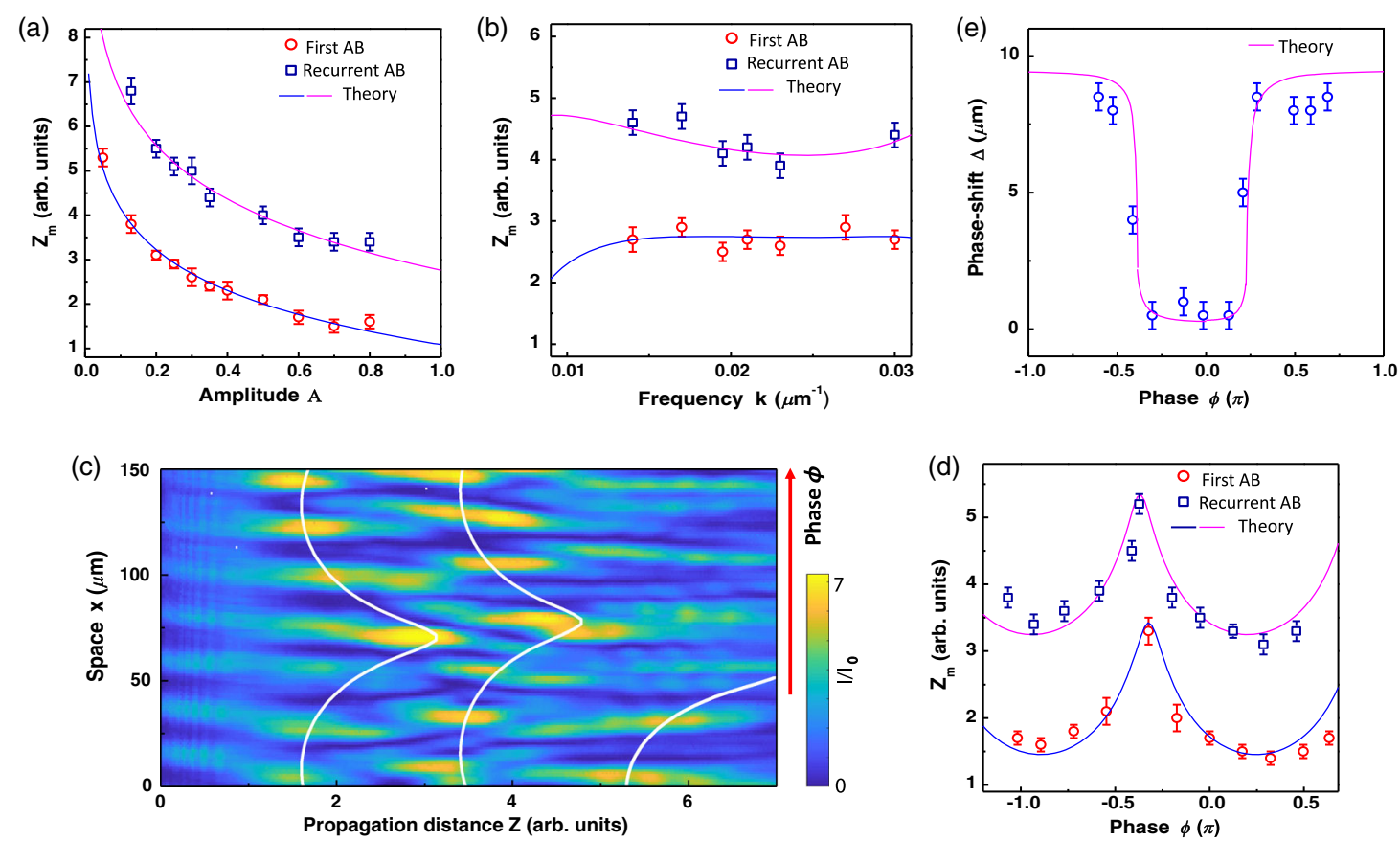

FIG. 3. Properties of the recurrent behavior. Recurrence partial period measured (dots) by varying (a) the amplitude of the input excitation $\left(k=0.023 \mu \mathrm{m}^{-1}\right)$ and (b) the frequency of the input mode $(A=0.3)$. (c) Evolving intensity distribution detected for an input phase that varies along $x\left(k=0.030 \mu \mathrm{m}^{-1}\right)$. White lines interpolate local maxima and serve as guides. (d) $Z_{m}$ as a function of the initial dephasing. Blue and magenta lines in panels (a)-(d) are fitting functions according to Eq. (3) (see the Appendix). (e) Recurrence phase shift varying the input phase: measured sharp transitions (dots) and predicted behavior (line).

(a)

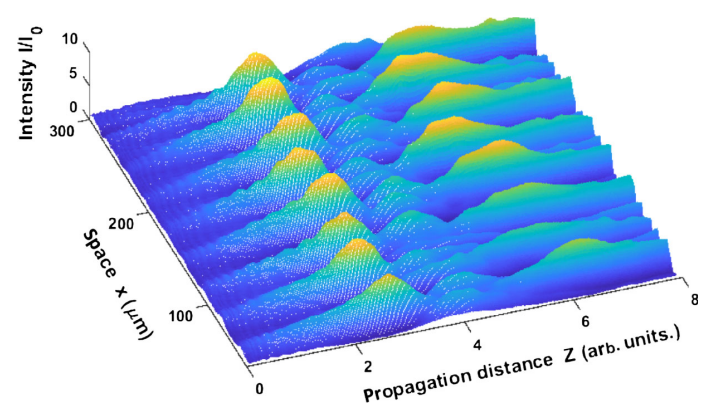

(c)

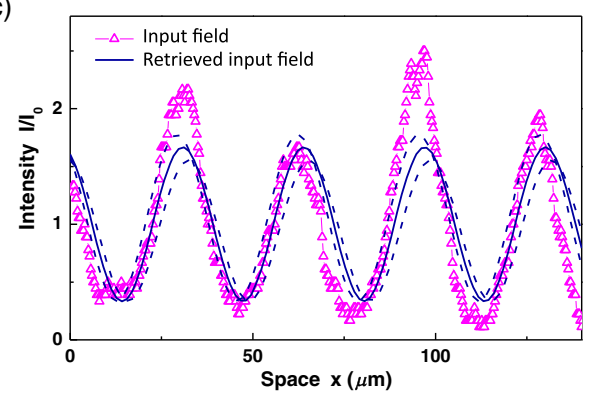

(b)

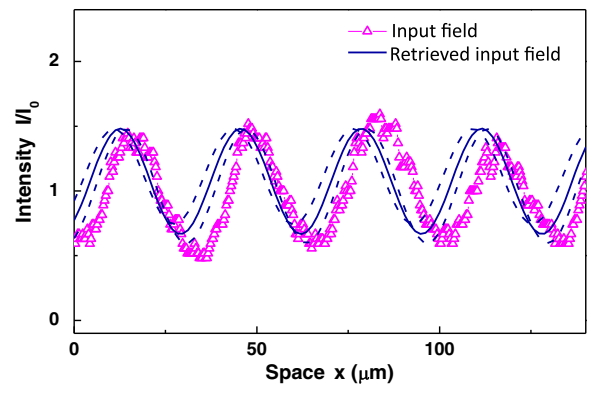

(d)

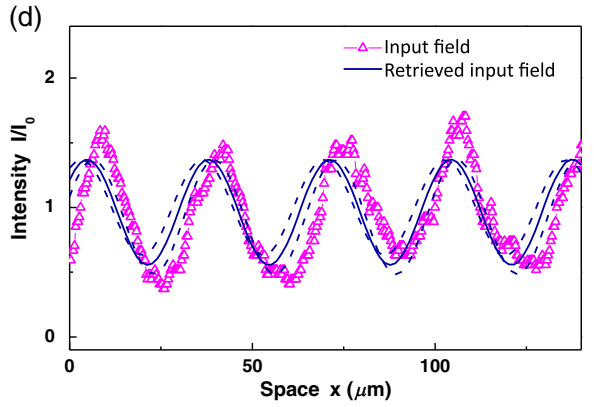

FIG. 4. Inverse problem. (a) Experimental observation used to test the predictability of the input state from the recurrent dynamics $\left(k=0.021 \mu \mathrm{m}^{-1}\right)$. We show measured (dots) and retrieved (line) input fields for different initial phases: (b) $\phi \simeq \pi$, (c) $\phi \simeq 0$, and (d) $\phi \simeq 0.3 \pi$. Dashed lines indicate the uncertainty of the retrieved condition. 
obtain the theoretical parameter $\vartheta \simeq 0.15$, consistent with the one extracted from the $\mathrm{AB}$ profile. Moreover, a sharp transition is expected for the recurrent phase shift as a function of the input phases [16]. In Fig. 3(e), we report the measured shift, which sharply passes from $\Delta \approx 0$ to $\Delta \approx$ $1 / 2 k$ by varying the phase delay, a behavior that agrees well with the theoretical condition $\cos (\phi) \gtrless \cos (2 \vartheta)$. These effects indicate that the coherence of the field is maintained as energy is exchanged between different modes: Phase locking dominates the nonlinear stage of the unstable dynamics, and thermalization slows down.

The deterministic properties of the return cycle imply its predictability once the input condition is completely known, and vice versa. To investigate this integrable character in experimental conditions, we retrieve the actual initial state from the features exhibited by the recurrent stage (inverse problem). We consider the FPUT dynamics reported in Fig. 4(a). The phase $B$ of the input perturbation is obtained by taking into account that the periodic transverse position $X_{1}$ at which the first $\mathrm{AB}$ has its maximum intensity strictly depends on $\phi$, as well as the specific shift $\Delta$ characterizing the return cycle. In fact, according to Eq. (5), we have $X_{1}=(\arg (\alpha)-\vartheta+\pi / 2) / k$. At each recurrent cycle, the breather solution is transversely shifted by $\Delta=\arg (\alpha \beta) / k$. Therefore, when $\vartheta \approx 0, \arg \beta \simeq$ $k\left(\Delta-X_{1}\right)+\pi / 2$, and the phase of the input excitation can be evaluated as

$$
B=\arg (\gamma) \approx k\left(\Delta-X_{1}\right)-\vartheta,
$$

where $k$ and $\vartheta$ are extracted from the first $\mathrm{AB}$ profile. The amplitude of the single mode follows from the observed $Z_{1}$ through the scaling in Fig. 3(a). As shown in Figs. 4(b)-4(d) for different initial dephasing, the field retrieved using this procedure agrees well with the experimental input condition that generates the recurrence: The nonequilibrium dynamics can be accurately traced on the basis of the underlying integrable model.

\section{DISCUSSION AND CONCLUSION}

The predictability of the FPUT dynamics is a general property of the system and does not depend on the specific input state. However, nonlinear evolution becomes more complex when several harmonics are initially excited. We observe that, for two excited input modes, recurrent highintensity patterns still occur, but their periodicity is lost and different states are experienced during propagation. Starting with a superposition of a large number of modes, random noise, or localized perturbations, wave turbulence sets in $[43,44]$. In these complex regimes, disordered nonlinear interactions may play a crucial role with respect to exact solutions of the underlying model [45]. Finally, we note that the observed recurrence gradually disappears as the external pump is weakened, a finding that further corroborates integrability as the basis of the phenomenon.

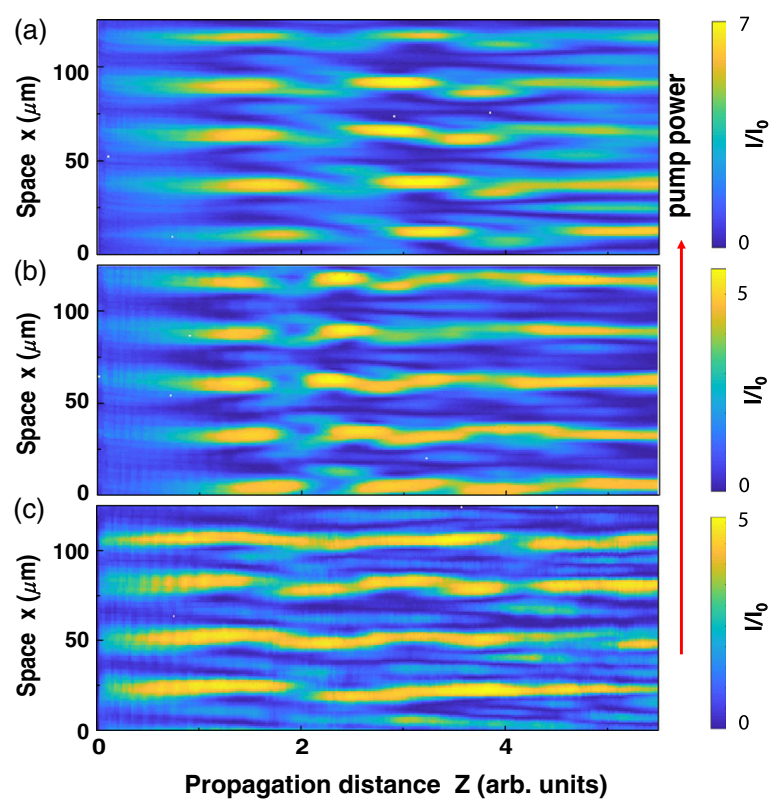

FIG. 5. From the integrable to the nonintegrable regime. We show the nonlinear evolution detected for $k=0.021 \mu \mathrm{m}^{-1}$ and $A=0.5$ by varying the external pump power: (a) $P=6 \mathrm{~mW}$, (b) $P=2 \mathrm{~mW}$, and (c) $P=0.5 \mathrm{~mW}$. The recurrent behavior in panel (a) is superseded by the appearance of spatial solitons (c) as the dynamics is far from integrability (highly saturated conditions).

The continuous transition towards the nonintegrable regime is reported in Fig. 5. Pseudorecurrent breather structures persist as the nonlinearity approaches the saturable regime and the model departs from the canonical NLSE [46] [Figs. 5(a) and 5(b)], whereas no return to the initial state occurs in highly saturated, nonintegrable conditions [Fig. 5(c)]. Here, interacting spatial solitons form and evolve towards equilibrium, compatibly with a soliton turbulence scenario [47].

In conclusion, we have observed the Fermi-Pasta-UlamTsingou recurrence in nonlinear spatial optics, providing unprecedented experimental evidence of its underlying integrable dynamics. We reveal that the single-mode input field deterministically sets the properties of the recurrent behavior for several breathing cycles, in close agreement with the analytic NLSE theory. The dynamics is thus accurately predicted, a result that extends predictive approaches to unstable wave regimes and maps a strategy to achieve the control of localized large-amplitude waves in environmental conditions. The optical setting we have introduced, in which the input condition can, in principle, be arbitrarily shaped, provides a general test bed for investigating universal nonlinear phenomena. Our findings shed light on the foundations of the FPUT problem and represent a unique test for nonlinear wave theory, with broad implications in hydrodynamics, nonlinear optics, Bose-Einstein condensates, and beyond. 


\section{ACKNOWLEDGMENTS}

We acknowledge funding from Sapienza Ateneo 2016 and Sapienza Ateneo 2017 and the Lazio Innova 2016 project. L.Z. and D. P. received funding from the Natural Science Foundation of China (Grant No. 61505116) and China Postdoctoral Science Foundation (No. 2018M630978). C. C. acknowledges support from the John Templeton Foundation (No. 58277), QuantERA ERA-NET Co-fund (Grant No. 731473, project QUOMPLEX), and PRIN NEMO 2015. A. J. A. acknowledges the support of the Peter Brojde Center for Innovative Engineering. P. G. G. acknowledges support from the Russian Science Foundation Grant No. 18-11-00316.

D. P., M. F., L.Z. contributed equally to this work.

\section{APPENDIX: METHODS}

\section{Experimental setup}

A $y$-polarized optical beam at wavelength $\lambda=532 \mathrm{~nm}$ from a continuous 30-mW Nd: YAG laser source is split and recombined in the $x z$ plane to form a symmetric three-wave interferometer, with the two arms having opposite wave vectors and forming an angle $\theta$ with the $300-\mu \mathrm{W}$ central beam. A sketch of the optical system is shown in Fig. 1(a). The interference pattern is focused by a cylindrical lens down to a quasi-one-dimensional beam with waist $\omega_{0}=15 \mu \mathrm{m}$ along the $y$ direction and periodically modulated along the $x$ direction [inset in Fig. 1(a)]. The copropagating waves are launched into an optical quality specimen of $2.1^{(x)} \times 1.9^{(y)} \times$ $2.5^{(z)} \mathrm{mm} \mathrm{K}_{0.964} \mathrm{Li}_{0.036} \mathrm{Ta}_{0.60} \mathrm{Nb}_{0.40} \mathrm{O}_{3}$ (KLTN) with $\mathrm{Cu}$ and $\mathrm{V}$ impurities. The crystal exhibits a ferroelectric phase transition at the Curie temperature $T_{C}=284 \mathrm{~K}$. Nonlinear light dynamics are studied in the paraelectric phase at $T=T_{C}+8 \mathrm{~K}$, a condition ensuring a large nonlinear response and a negligible effect of small-scale disorder [48]. The time-dependent photorefractive response sets in when an external bias field $E$ is applied along $y$ (voltage $V=500 \mathrm{~V}$ ). To have a so-called Kerr-like (cubic) nonlinearity from the photorefractive effect, the crystal is continuously pumped with an $x$-polarized $15-\mathrm{mW}$ laser at $\lambda=633 \mathrm{~nm}$. The pump does not interact with the principal beams propagating along the $z$ axis and only constitutes a reference intensity larger than the single-mode perturbed background wave. The spatial intensity distribution is measured at the crystal output as a function of the exposure time by means of a high-resolution imaging system composed of an objective lens $(N A=0.5)$ and a CCD camera at $15 \mathrm{~Hz}$.

\section{Beam propagation in photorefractive media}

Generally, nonlinear optical wave propagation in paraxial conditions is described by the parabolic wave equation $i \partial_{z} A+(1 / 2 k) \nabla^{2} A+(k / n) \delta n A=0$, where $A(x, y, z)$ is the slowly varying optical-field envelope normalized to a reference intensity, $k=2 \pi n / \lambda$, and $n$ the index of refraction. The self-interaction term $\delta n=\delta n\left(|A|^{2}\right)$ accounts for the nonlinear response of the supporting medium and, in the case of a photorefractive nonlinearity, it also depends parametrically on time, $\delta n=\delta n\left(|A|^{2}, t\right)$ [38]. In fact, as the light beam impinges on the biased photorefractive crystal, it starts to generate an illumination-dependent variation of the index of refraction by means of carriers' excitations and their spatial redistribution. The amplitude of the resulting nonlinear change, on average, grows with the exposure time up to a saturation value. This process occurs on a slow timescale, typically seconds for peak intensities of a few $\mathrm{kW} \mathrm{cm}{ }^{-2}$ [38]. In our system, the dependence on time can be factored out in a first approximation, $\delta n=f(t) \Delta n\left(|A|^{2}\right)$, so that the focusing photorefractive nonlinearity in centrosymmetric media reads as $\delta n=f(t) \Delta n_{0} /\left(1+|A|^{2}\right)^{2}$, where $\Delta n_{0}$ includes the quadratic electro-optic effect. For $|A|^{2} \ll 1$, as occurs in our externally pumped configuration, we obtain the Kerr-like regime where $\Delta n\left(|A|^{2}\right) \approx 2 \Delta n_{0}|A|^{2}$, apart from a constant shift. The dynamics along each transverse coordinate is thus described by the one-dimensional equation $i \partial_{z} A+(1 / 2 k) \partial_{x x}^{2} A+2 \rho|A|^{2} A=0$, where $\rho=$ $(k / n) \Delta n_{0} f(t)$ is the single parameter that fixes the relative strength of diffraction and nonlinearity. Introducing the following change of variables, $Z=\rho z, X=\sqrt{2 k \rho} x$ and $A(x, z)=\psi(X, Z)$, the equation can be transformed into the dimensionless NLSE [Eq. (1)]: $i \partial_{Z} \psi+\partial_{X X} \psi+$ $2|\psi|^{2} \psi=0$. An analogous renormalization of the wave equation is widely adopted in nonlinear fiber optics, where the optical power of the input wave is exploited to mimic the spatial dynamics along the fiber [49]. Rigorously, since the normalization makes dispersive terms slowly varying along the propagation, the approach allows us to observe only an effective field evolution.

In the present case, the evolution in $Z$ is studied at a fixed value of $z$ (the crystal output) by varying the exposure time $t$. In fact, experimental results obtained in similar photorefractive KTN crystals have verified that the average index change grows and saturates according to $f(t)=$ $1-\exp (-t / \tau)$ [50]. The time dependence is well defined through the saturation time $\tau$ once the input beam intensity, applied voltage, and temperature have been fixed. Using this relation with the measured $\tau \approx 80 \mathrm{~s}$, observations at the crystal output are rescaled as a function of the effective distance $Z$. The nonlinear response function $f(t)$ represents the main limitation of the technique in reconstructing the spatial dynamics from time-resolved measurements. Specifically, $f(t)$ is independent of the local intensity only in a first approximation, a fact that affects the accuracy of the obtained field evolution. When the intensity distribution presents large intensity variations or strong spatial inhomogeneities, intensity-dependent corrections to $f(t)$ should be taken into account to have a quantitative reconstruction along the evolution coordinate. These high-order terms are nonlocal in space and time; their main effect is that the time 
evolution of high-intensity regions slows down [38]. Therefore, in the present case, the method is particularly accurate up to a distance $Z_{1}$ (first-appearance time). Small longitudinal deformations appear at longer evolution scales [Figs. 2(a) and 2(b)], and the relative distance between the observed $\mathrm{AB}$ structures cannot be accurately evaluated. This fact explains the discrepancy with theory for the value of the recurrent period when measured through the $Z$ distance between returning intensity maxima. In particular, the recurrent $A B$ seems to appear at an effective distance that is always shorter than expected according to theoretical predictions. Moreover, as the nonlinearity finally saturates in time, the field dynamics at large $Z$ departs from that of the integrable model, and evolution towards thermalization is observed.

\section{Analysis of the recurrence}

The intensity traces detected as the normalized distribution $I / I_{0}=|\psi|^{2}$ reaches their maxima are compared with the Akhmediev breather profile in Eq. (4). In fitting the data in Figs. 2(c)-(d), the coefficients $\xi_{m}, k, \vartheta$, and $X_{m}$ are considered as bounded parameters. The detected recurrence partial period is analyzed according to Eq. (3); for the measurements in Fig. 3(a), we consider $a \log (b / \varepsilon)$ as a fitting function, with $a$ and $b$ free parameters. In Fig. 3(b), we use $a \log \left(b\left(k \sqrt{K_{\max }^{2}-k^{2}}\right)^{2}\right) / k \sqrt{K_{\max }^{2}-k^{2}}$, where $a$ and $b$ are free parameters and $K_{\max } \approx 0.03 \mu \mathrm{m}^{-1}$ is the wave vector with maximum gain that we independently measure from spontaneous $\mathrm{MI}$ of the background wave. In Fig. 3(d), the detected $Z_{m}$ is compared to $\log \left(a / \sqrt{c_{1}^{2}+c_{2}^{2}-2 c_{1} c_{2} \cos (\phi+b)}\right)$, with $a, b, c_{1}$, and $c_{2}$ being bounded parameters. In this case, it is interesting to note that the fitting procedure returns $c_{1} \simeq c_{2}$, that is, a balanced condition for the interferometer arms, as settled in the experiment. In Fig. 3(e), the fitting functions are $a+b /[\cos (\phi)-\cos (c)]$, as predicted for the symmetric case $\left|c_{1}\right| \approx\left|c_{2}\right|$. As for the retrieval of the input perturbation, from the observed recurrences in Figs. 4(a) and $4(\mathrm{~b})$, we measure, for example, $k=0.021 \mu \mathrm{m}^{-1}$, $\Delta=1 \pm 1 \mu \mathrm{m}, Z_{1}^{\prime}=2.6 \pm 0.1, \vartheta=0.1$, which, for $X_{1}=$ $208 \mu \mathrm{m}$, gives $B=-40.1 \pm 0.3$ and $A=0.33 \pm 0.02$. For comparison, $B=-40.9 \pm 0.2$ and $A=0.34 \pm 0.01$ are the values obtained by fitting the experimental initial intensity.

[1] T. Dauxois, Fermi, Pasta, Ulam, and a Mysterious Lady, Phys. Today 61 No. 1, 55 (2008).

[2] J. Ford, The Fermi-Pasta-Ulam Problem: Paradox Turns Discovery, Phys. Rep. 213, 271 (1992).

[3] The Fermi-Pasta-Ulam Problem: A Status Report, edited by G. Gallavotti, Lecture Notes in Physics (Springer, Berlin, Heidelberg, 2008), Vol. 728, doi: 10.1007/978-3-54072995-2.
[4] G. P. Berman and F. M. Izrailev, The Fermi-Pasta-Ulam Problem: Fifty Years of Progress, Chaos 15, 015104 (2005).

[5] G. Benettin, H. Christodoulidi, and A.Ponno, The FermiPasta-Ulam Problem and Its Underlying Integrable Dynamics, J. Stat. Phys. 152, 195 (2013).

[6] M. Onorato, L. Vozella, D. Proment, and Y. V. Lvov, Route to Thermalization in the $\alpha$-Fermi-Pasta-Ulam System, Proc. Natl. Acad. Sci. U.S.A. 112, 4208 (2015).

[7] M. Guasoni, J. Garnier, B. Rumpf, D. Sugny, J. Fatome, F. Amrani, G. Millot, and A. Picozzi, Incoherent Fermi-PastaUlam Recurrences and Unconstrained Thermalization Mediated by Strong Phase Correlations, Phys. Rev. X 7, 011025 (2017).

[8] Y. V. Lvov and M. Onorato, Double Scaling in the Relaxation Time in the $\beta$-Fermi-Pasta-Ulam-Tsingou Model, Phys. Rev. Lett. 120, 144301 (2018).

[9] S. Wabnitz and B. Wetzel, Instability and Noise-induced Thermalization of Fermi-Pasta-Ulam Recurrence in the Nonlinear Schrodinger Equation, Phys. Lett. A 378, 2750 (2014).

[10] O. Kimmoun, H. C. Hsu, H. Branger, M. S. Li, Y. Y. Chen, C. Kharif, M. Onorato, E. J. R. Kelleher, B. Kibler, N. Akhmediev, and A. Chabchoub, Modulation Instability and Phase-Shifted Fermi-Pasta-Ulam Recurrence, Sci. Rep. 6, 28516 (2016).

[11] S. Trillo, G. Deng, G. Biondini, M. Klein, G. F. Clauss, A. Chabchoub, and M. Onorato, Experimental Observation and Theoretical Description of Multisoliton Fission in Shallow Water, Phys. Rev. Lett. 117, 144102 (2016).

[12] M. Wu and C.E. Patton, Experimental Observation of Fermi-Pasta-Ulam Recurrence in a Nonlinear Feedback Ring System, Phys. Rev. Lett. 98, 047202 (2007).

[13] C. Bao, J. A. Jaramillo-Villegas, Y. Xuan, D. E. Leaird, M. Qi, and A. M. Weiner, Observation of Fermi-PastaUlam Recurrence Induced by Breather Solitons in an Optical Microresonator, Phys. Rev. Lett. 117, 163901 (2016).

[14] G. Simaeys, P. Emplit, and M. Haelterman, Experimental Demonstration of the Fermi-Pasta-Ulam Recurrence in a Modulationally Unstable Optical Wave, Phys. Rev. Lett. 87, 033902 (2001).

[15] A. Mussot, A. Kudlinski, M. Droques, P. Szriftgiser, and N. Akhmediev, Fermi-Pasta-Ulam Recurrence in Nonlinear Fiber Optics: The Role of Reversible and Irreversible Losses, Phys. Rev. X 4, 011054 (2014).

[16] A. Mussot, C. Naveau, M. Conforti, A. Kudlinski, F. Copie, P. Szriftgiser, and S. Trillo, Fibre Multi-wave Mixing Combs Reveal the Broken Symmetry of Fermi-Pasta-Ulam Recurrence, Nat. Photonics 12, 303 (2018).

[17] M. J. Ablowitz, B. Prinari, and A. D. Trubatch, Discrete and Continuous Nonlinear Schrodinger Systems, London Mathematical Society (Cambridge University Press, Cambridge, England, 2004), Vol. 302.

[18] J. M. Dudley, G. Genty, F. Dias, B. Kibler, and N. Akhmediev, Modulation Instability, Akhmediev Breathers and Continuous Wave Supercontinuum Generation, Opt. Express 17, 21497 (2009). 
[19] B. Kibler, J. Fatome, C. Finot, G. Millot, F. Dias, G. Genty, N. Akhmediev, and J. M. Dudley, The Peregrine Soliton in Nonlinear Fibre Optics, Nat. Phys. 6, 790 (2010).

[20] B. Frisquet, B. Kibler, and G. Millot, Collision of Akhmediev Breathers in Nonlinear Fiber Optics, Phys. Rev. X 3, 041032 (2013).

[21] M. Erkintalo, K. Hammani, B. Kibler, C. Finot, N. Akhmediev, J. M. Dudley, and G. Genty, Higher-Order Modulation Instability in Nonlinear Fiber Optics, Phys. Rev. Lett. 107, 253901 (2011).

[22] A. Chabchoub, N. P. Hoffmann, and N. Akhmediev, Rogue Wave Observation in a Water Wave Tank, Phys. Rev. Lett. 106, 204502 (2011).

[23] A. Chabchoub, N. Hoffmann, M. Onorato, and N. Akhmediev, Super Rogue Waves: Observation of a Higher-Order Breather in Water Waves, Phys. Rev. X 2 , 011015 (2012).

[24] B. Kibler, A. Chabchoub, A. Gelash, N. Akhmediev, and V.E. Zakharov, Super-regular Breathers in Optics and Hydrodynamics: Omnipresent Modulation Instability Beyond Simple Periodicity, Phys. Rev. X 5, 041026 (2015).

[25] M. Onorato, S. Residori, U. Bortolozzo, A. Montina, and F. T. Arecchi, Rogue Waves and Their Generating Mechanisms in Different Physical Contexts, Phys. Rep. 528, 47 (2013).

[26] J. M. Dudley, F. Dias, M. Erkintalo, and G. Genty, Instabilities, Breathers and Rogue Waves in Optics, Nat. Photonics 8, 755 (2014).

[27] N. Akhmediev et al., Roadmap on Optical Rogue Waves and Extreme Events, J. Opt. (Paris) 18, 063001 (2016).

[28] D. Pierangeli, F. Di Mei, C. Conti, A. J. Agranat, and E. DelRe, Spatial Rogue Waves in Photorefractive Ferroelectrics, Phys. Rev. Lett. 115, 093901 (2015).

[29] D. Pierangeli, G. Musarra, F. Di Mei, G. Di Domenico, A. J. Agranat, C. Conti, and E. DelRe, Enhancing Optical Extreme Events through Input Wave Disorder, Phys. Rev. A 94, 063833 (2016).

[30] P. Suret, R. El Koussaifi, A. Tikan, C. Evain, S. Randoux, C. Szwaj, and S. Bielawski, Single-Shot Observation of Optical Rogue Waves in Integrable Turbulence Using Time Microscopy, Nat. Commun. 7, 13136 (2016).

[31] E. Infeld, Quantitive Theory of the Fermi-Pasta-Ulam Recurrence in the Nonlinear Schrodinger Equation, Phys. Rev. Lett. 47, 717 (1981).

[32] P. Berman and A. R. Kolovsky, The Limit of Stochasticity for a One-Dimensional Chain of Interacting Oscillators, Sov. Phys. JETP 60, 1116 (1984).

[33] P. G. Grinevich and P. M. Santini, The Finite Gap Method and the Analytic Description of the Exact Rogue Wave Recurrence in the Periodic NLS Cauchy Problem, Nonlinearity 31, 5258 (2018).

[34] P. G. Grinevich and P. M. Santini, The Exact Rogue Wave Recurrence in the NLS Periodic Setting via Matched Asymptotic Expansions, for 1 and 2 Unstable Modes, Phys. Lett. A 382, 973 (2018).
[35] N. Akhmediev, V. Eleonskii, and N. Kulagin, Exact FirstOrder Solutions of the Non-linear Schrodinger Equation, Theor. Math. Phys. 72, 809 (1987).

[36] F. DiMei, P. Caramazza, D. Pierangeli, G. Di Domenico, H. Ilan, A. J. Agranat, P. Di Porto, and E. DelRe, Intrinsic Negative Mass from Nonlinearity, Phys. Rev. Lett. 116, 153902 (2016).

[37] C. Barsi, W. Wan, and J. W. Fleischer, Imaging through Nonlinear Media Using Digital Holography, Nat. Photonics 3, 211 (2009).

[38] E. DelRe, B. Crosignani, and P. Di Porto, Photorefractive Solitons and Their Underlying Nonlocal Physics, Prog. Opt. 53, 153 (2009).

[39] S. A. Chin, O. A. Ashour, and M. R. Belic, Anatomy of the Akhmediev Breather: Cascading Instability, First Formation Time, and Fermi-Pasta-Ulam Recurrence, Phys. Rev. E 92, 063202 (2015).

[40] J. M. Martinis, S. Nam, J. Aumentado, and C. Urbina, Rabi Oscillations in a Large Josephson-Junction Qubit, Phys. Rev. Lett. 89, 117901 (2002).

[41] N. Devine, A. Ankiewicz, G. Genty, J. M. Dudley, and N. Akhmediev, Recurrence Phase Shift in Fermi-Pasta-Ulam Nonlinear Dynamics, Phys. Lett. A 375, 4158 (2011).

[42] J. M. Soto-Crespo, N. Devine, and N. Akhmediev, Adiabatic Transformation of Continuous Waves into Trains of Pulses, Phys. Rev. A 96, 023825 (2017).

[43] A. Picozzi, J. Garnier, T. Hansson, P. Suret, S. Randoux, G. Millot, and D. N. Christodoulides, Optical Wave Turbulence: Towards a Unified Nonequilibrium Thermodynamic Formulation of Statistical Nonlinear Optics, Phys. Rep. 542, 1 (2014).

[44] D. Pierangeli, F. Di Mei, G. Di Domenico, A. J. Agranat, C. Conti, and E. DelRe, Turbulent Transitions in Optical Wave Propagation, Phys. Rev. Lett. 117, 183902 (2016).

[45] D. Pierangeli, A. Tavani, F. Di Mei, A. J. Agranat, C. Conti, and E. DelRe, Observation of Replica Symmetry Breaking in Disordered Nonlinear Wave Propagation, Nat. Commun. 8, 1501 (2017).

[46] N. N. Akhmediev, D. R. Heatley, G. I. Stegeman, and E. M. Wright, Pseudorecurrence in Two-Dimensional Modulation Instability with a Saturable Self-Focusing Nonlinearity, Phys. Rev. Lett. 65, 1423 (1990).

[47] B. Rumpf and A.C. Newell, Coherent Structures and Entropy in Constrained, Modulationally Unstable, Nonintegrable Systems, Phys. Rev. Lett. 87, 054102 (2001).

[48] D. Pierangeli, M. Ferraro, F. Di Mei, G. Di Domenico, C. E. M. de Oliveira, A. J. Agranat, and E. DelRe, Super-Crystals in Composite Ferroelectrics, Nat. Commun. 7, 10674 (2016).

[49] J. Fatome, C. Finot, G. Millot, A. Armaroli, and S. Trillo, Observation of Optical Undular Bores in Multiple FourWave Mixing, Phys. Rev. X 4, 021022 (2014).

[50] Q. Lu, J. Han, H. Dai, B. Ge, and S. Zhao, Visualization of Spatial-Temporal Evolution of Light-Induced Refractive Index in Mn:Fe:KTN Co-Doped Crystal Based on Digital Holographic Interferometry, IEEE Photonics J. 7, 2600711 (2015). 